\title{
Theta Stimulation Polymerizes Actin in Dendritic Spines of Hippocampus
}

\author{
Bin Lin, ${ }^{1}$ Enikö A. Kramár, ${ }^{1}$ Xiaoning Bi, ${ }^{1}$ Fernando A. Brucher, ${ }^{1}$ Christine M. Gall, ${ }^{2}$ and Gary Lynch ${ }^{1}$ \\ ${ }^{1}$ Department of Psychiatry and Human Behavior, University of California, Irvine, California 92617-1695, and 2Department of Anatomy and Neurobiology, \\ University of California, Irvine, California 92697-4292
}

It has been proposed that the endurance of long-term potentiation (LTP) depends on structural changes entailing reorganization of the spine actin cytoskeleton. The present study used a new technique involving intracellular and extracellular application of rhodaminephalloidin to conventional hippocampal slices to test whether induction of LTP by naturalistic patterns of afferent activity selectively increases actin polymerization in juvenile to young adult spines. Rhodamine-phalloidin, which selectively binds to polymerized actin, was detected in perikarya and proximal dendrites of CA1 pyramidal cells that received low-frequency afferent activity but was essentially absent in spines and fine dendritic processes. Theta pattern stimulation induced LTP and caused a large (threefold), reliable increase in labeled spines and spine-like puncta in the proximal dendritic zone containing potentiated synapses. The spines frequently occurred in the absence of labeling to other structures but were also found in association with fluorescent dendritic processes. These effects were replicated ( $>10$-fold increase in labeled spines) using extracellular applications of rhodamine-phalloidin. Increases in labeling appeared within $2 \mathrm{~min}$, were completely blocked by treatments that prevent LTP induction, and occurred in slices prepared from young adult rats. These results indicate that near-threshold conditions for inducing stable potentiation cause the rapid polymerization of actin in mature spines and suggest that the effect is both sufficiently discrete to satisfy the synapse-specificity rule of LTP as well as rapid enough to participate in the initial stages of LTP consolidation.

Key words: CA1; hippocampus; LTP; actin; phalloidin; plasticity; whole-cell recording

\section{Introduction}

The idea that changes in spine morphology are responsible for the remarkable stability of long-term potentiation (LTP) has an extended history. An initial experiment found that high-frequency stimulation of the entorhinal cortex caused spine swelling throughout the dentate gyrus (Van Harreveld and Fifkova, 1975), but the first studies using brief stimulation bursts described changes in the variability of spine and postsynaptic density (PSD) measures in field CA1 (Lee et al., 1980). This was interpreted as evidence that inducing LTP causes elongated spines to become rounder. Subsequent electron microscopic studies provided additional evidence that rounding of spines occurs within minutes of LTP induction in field CA1 (Chang and Greenough, 1984) and that LTP-related changes in spine shape also occur in the dentate gyrus (Desmond and Levy, 1986). More recent work suggests that the triggering events for LTP can, at least under some conditions, produce more dramatic changes, including the creation of new spines (Yuste and Bonhoeffer, 2001) or splitting of extant ones (Geinisman et al., 1991; Toni et al., 1999; Yuste and Bonhoeffer,

Received 0ct. 14, 2004; revised Jan. 17, 2005; accepted Jan. 18, 2005.

This research was supported by Grant MH16007 from the National Institute of Mental Health and Grant NS37799 from the National Institute of Neurological Disorders and Stroke. We thank Yue Qin Yao for excellent technical assistance.

Correspondence should be addressed to Christine M. Gall, Department of Anatomy and Neurobiology, University of California, Irvine, Gillespie Neuroscience Research Facility Room 3226, Irvine, CA 92697-4292. E-mail: cmgall@uci.edu.

D01:10.1523/JNEUROSCI.4283-04.2005

Copyright $\odot 2005$ Society for Neuroscience $\quad$ 0270-6474/05/252062-08\$15.00/0
2001; Fiala et al., 2002). Moreover, the activity-driven change from an elongated to a round configuration, as inferred from electron microscopic studies, has been visualized in field CA1 of cultured hippocampal slices using recently introduced optical techniques (Matsuzaki et al., 2004).

Activity-dependent modifications to spine anatomy presumably involve some degree of cytoskeletal reorganization, and there are clear demonstrations that stimulation of glutamate receptors, as well as intense afferent activity, modifies the actin network in spines of dissociated hippocampal neurons (Fischer et al., 1998; Matus, 2000; Ackermann and Matus, 2003) and in organotypic cultures of hippocampus (Okamoto et al., 2004). Pertinent to this, LTP fails to consolidate in adult hippocampal slices treated with agents that block actin polymerization (Krucker et al., 2000; Fukazawa et al., 2003), and potentiation in the perforant path projections leads to an increase in F-actin labeling across the full mediolateral extent of the outer molecular layer of the dentate gyrus (Fukazawa et al., 2003). In the experiments reported here, we used a new technique to further investigate whether polymerization chiefly relates to activity-driven spine maturation or is also a concomitant of synaptic plasticity in adult brain. If the latter, then polymerization should be triggered in fully developed synapses by naturalistic patterns of afferent activity that are near threshold for inducing LTP. We also investigated whether the effect is (1) selective enough (i.e., occurs in sparse populations of spines and does not affect whole dendritic segments) to satisfy the LTP synapse-specificity rule (Bliss and Lomo, 1973) and (2) rapid 
enough to contribute to the component of LTP consolidation that begins within the first minute or two after induction (Barrionuevo et al., 1980; Lynch et al., 1991; Larson et al., 1993; Huang et al., 1999; Chun et al., 2001).

\section{Materials and Methods}

All animal procedures were conducted in accordance with the National Institutes of Health Guide for the Care and Use of Laboratory Animals and with protocols approved by the Institutional Animal Care and Use Committee of the University of California at Irvine (Irvine, CA). Efforts were made to minimize animal suffering and numbers of rats used in the work described.

Intracellular application of rhodamine-phalloidin. Hippocampal slices (350 $\mu \mathrm{m}$ thick) were prepared from 15- to 25-d-old male Sprague Dawley rats (Charles River Laboratories, Wilmington, MA) and placed in a holding chamber for at least $1 \mathrm{~h}$ before being transferred to a recording chamber; in both instances, slices were submerged in artificial CSF (ACSF) oxygenated with $95 \% \mathrm{O}_{2} / 5 \% \mathrm{CO}_{2}$ and containing the following (in mM): $124 \mathrm{NaCl}, 3 \mathrm{KCl}, 1.25 \mathrm{KH}_{2} \mathrm{PO}_{4}, 3.4 \mathrm{CaCl}_{2}, 2.5 \mathrm{MgSO}_{4}, 26$ $\mathrm{NaHCO}_{3}$, and $10 \mathrm{D}$-glucose, $\mathrm{pH}$ 7.3. In the recording chamber, ACSF was infused at $1.2 \mathrm{ml} / \mathrm{min}$, and experiments were performed at $32^{\circ} \mathrm{C}$. Unless otherwise noted, chemicals were purchased from Sigma (St. Louis, MO).

CA1 pyramidal neurons were visualized using an infrared microscope (BX50WI; Olympus, Melville, NY) and differential interference contrast configuration. Whole-cell recordings were made with 3-5 $\mathrm{M} \Omega$ recording pipettes containing the following (in $\mathrm{mm}$ ): $130 \mathrm{Cs}$ gluconate, $10 \mathrm{CsCl}, 0.2$ EGTA, $8 \mathrm{NaCl}$, 2 ATP, $0.3 \mathrm{GTP}$, and 10 HEPES, pH 7.35, 290-300 mOsm. Rhodamine-phalloidin was added to the pipette solution at a concentration $(6 \mu \mathrm{M})$ that in pilot studies did not interfere with baseline physiology or the induction of LTP. Holding potentials were set to -70 $\mathrm{mV}$ after correcting for the junction potential. EPSCs were recorded with a patch amplifier (AxoPatch-1D; Axon Instruments, Burlingame, CA) with a four-pole low-pass Bessel filter at $2 \mathrm{kHz}$ and digitized at $10 \mathrm{kHz}$. Two bipolar stimulating electrodes were placed in stratum (s.) radiatum $100-200 \mu \mathrm{m}$ above the readily visualized apical boundary of the pyramidal cell layer. The electrodes were placed on both sides of the recording site 200-300 $\mu \mathrm{m}$ from the clamp electrode. During baseline and posttheta burst recording periods, synaptic responses were evoked by stimulating the Schaffer collateral/commissural projections once every $20 \mathrm{~s}$. The stimulation intensity was adjusted to obtain EPSCs that were $<30 \%$ of the maximum response amplitude. Input and series resistances were

A

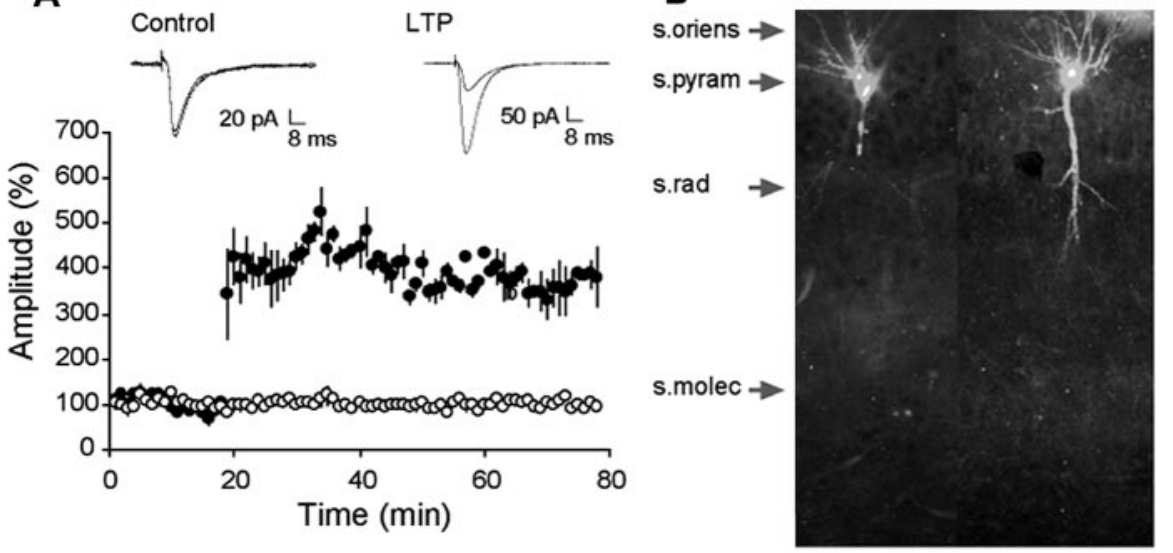

Figure 1. Recording with rhodamine-phalloidin-filled electrodes does not disrupt basic physiology or LTP. A, EPSCs elicited by stimulation of the Schaffer-commissural projections to the inner portion of stratum radiatum. Recordings were collected during the $20 \mathrm{~min}$ baseline period and for $60 \mathrm{~min}$ after TBS. The traces at the top show representative EPSCs collected from a control cell (no theta bursts; left) and from a cell in which LTP was induced by a single train of 10 theta bursts (right). Traces collected 60 min apart are superimposed in both cases. The graph in $\boldsymbol{A}$ summarizes results for a group of neurons that received control stimulation alone (open circles) or theta bursts at the $20 \mathrm{~min}$ time point (closed circles). Error bars represent SEM. B, Phalloidin-labeled cells from two control cases: laminar placements are indicated by arrows. s. pyram, Stratum pyramidale; s. rad, stratum radiatum; s. molec, stratum lacunosum-moleculare. Photomicrographs are at $10 \times$ objective magnification. continuously monitored and recordings were discarded if significant changes occurred.

Baseline responses were collected for 30 or $60 \mathrm{~min}$, after which theta burst stimulation (TBS), a $5 \mathrm{~Hz}$ train of 10 theta bursts, was applied through one of the two stimulation electrodes. Each burst contained four pulses at $100 \mathrm{~Hz}$, and the bursts were separated by $200 \mathrm{~ms}$. In this and subsequent groups, holding potentials were reduced to $0 \mathrm{mV}$ when TBS was applied and switched back to $-70 \mathrm{mV}$ afterward. Slices were fixed in $4 \%$ paraformaldehyde in $0.1 \mathrm{~m}$ phosphate buffer (PB), $\mathrm{pH} 7.4$, within 2 $\min (n=6)$ or $\geq 30(n=13)$ min after TBS. For control slices, baseline responses to stimulation pulses at three per minute were collected for 60-170 min, after which slices were fixed in $4 \%$ paraformaldehyde. Total recording time for the control and theta burst groups was equivalent.

Extracellular application of rhodamine-phalloidin. These studies used conventional hippocampal slices $(350 \mu \mathrm{m})$ prepared from young adult (30-42 d of age) male Sprague Dawley rats and maintained in an interface recording chamber perfused with preheated ACSF (Kramár et al., 2002). Field EPSPs (fEPSPs) were recorded from s. radiatum of CA1b using a single glass pipette $(2-3 \mathrm{M} \Omega)$. Orthodromic stimulation was delivered at two sites (CA1a and CA1c) in the apical branch of the Schaffer collateral-commissural projections. Pulses were administered in an alternating manner to the two electrodes at $0.05 \mathrm{~Hz}$ using a current that produced a response that was $50 \%$ of maximum. Rhodamine-phalloidin $(6 \mu \mathrm{M})$ was applied topically from a micropipette every $5 \mathrm{~min}$ for $20 \mathrm{~min}$. At the end of this period, a $5 \mathrm{~min}$ baseline was collected, after which a train of 10 theta bursts was delivered to one of the stimulation electrodes. This was repeated $2 \mathrm{~min}$ later to the second electrode. Slices were collected 1-2 min after the second stimulation episode. Slices in the control group received baseline stimulation for 3-4 min after the phalloidin treatment. Electrophysiological data were collected and digitized by NAC 2.0 Neurodata Acquisition System (Theta Burst, Irvine, CA) and stored on a disk.

Imaging. All slices were fixed in paraformaldehyde for $12-16 \mathrm{~h}$, cryoprotected in $20 \%$ sucrose/PB for $2 \mathrm{~h}$ at $4^{\circ} \mathrm{C}$, and then sectioned on a freezing microtome at a thickness of $30 \mu \mathrm{M}$ (parallel to the broad surface of the slice). Sections were collected into PB, mounted onto Superfrost slides (Fisher Scientific, Pittsburgh, PA), and then coverslipped with Vectashield (Vector Laboratories, Burlingame CA). Rhodaminephalloidin labeling was examined using epifluorescence illumination and either a Zeiss (Oberkochen, Germany) Axioscop microscope equipped with an Axiocam camera and AxioVision 3.1 software (for quantitative data collection) or, for additional qualitative analysis of labeled elements, a Zeiss Axiovert 2000 confocal microscope with laser scanning attachment (LSM 510 META). The wavelength used for rhodamine-phalloidin imaging was $543 \mathrm{~nm}$.

Measurements. Labeled spines and dendritic segments were measured and counted from a constant-sized sampling zone in the proximal s. radiatum between the two stimulating electrodes using locally developed software (a mix of Perl and C). Briefly, a constant-sized image segment was converted to 8 bits per pixel grayscale. Analysis was conducted blindly on batches of slices that had been sectioned and stained together; each batch contained nearequal numbers of experimental and control cases. The background was estimated as the average color of the whole image. A single threshold value for the entire batch was adjusted in trial runs so that objects corresponding in size to spines or dendritic branches were reliably counted. The pixel values for an image were normalized so that the overall background was a fixed gray value. This step reduced the impact of differences across the image in background intensity. The normalized image was then binarized using the single threshold established for 
all slices in a batch and then cleaned by "erosion" followed by "dilation" (for review, see Jain, 1989). Erosion removes black pixels that are not connected or only loosely connected to other black pixels and is implemented by taking each black pixel in the binarized image and counting the number of immediately surrounding black pixels (neighbors). If the number of neighbors is below a certain value, the pixel in question is deleted (made white). Dilation fills small gaps and smoothes out the boundaries of counted objects. Similarly as for erosion, we counted the number of surrounding black pixels for each white pixel in the image. If the number of black neighbors was above a constant, the center white pixel was turned black. After processing, objects were fitted by their best ellipse, and their eccentricity was calculated. Area and eccentricity were used to count objects as spines (small area, eccentricity $<2$ ), dendritic branches (eccentricity $>4$ ), or to reject observations as artifacts. The program printed out the final, processed images along with identification codes for the objects it categorized and counted. Several of these printouts per batch were compared with the original micrographs to check whether the target structures had been correctly identified.

\section{Results}

For intracellular application of rhodaminephalloidin studies, a total of 14 control (single pulse stimulation at three per minute) and 19 theta burst (10 $40 \mathrm{~ms}$ bursts separated by $200 \mathrm{~ms}$ ) experiments were conducted using whole-cell recording. Stimulation current during the train was maintained at the value used to elicit modest-sized, baseline EPSCs. In all cases, neurons were clamped with phalloidin-loaded electrodes and followed for over $1 \mathrm{~h}$ [controls, $97 \pm 48 \mathrm{~min}$ (mean $\pm \mathrm{SD}$ here and afterward); experimental, $101 \pm 50 \mathrm{~min}$ ].

Records collected with phalloidincontaining electrodes appeared normal, and, as shown in Figure $1 A$, recordings from a representative experiment did not show evident deterioration of responses throughout the recording session. LTP in field CA1 pyramidal neurons was of the same magnitude and stability in the phalloidin cases (Fig. $1 A$, bottom), as reported in previous whole-cell clamp studies (Arai et al., 1994; Lin et al., 2002), and its induction had no effect on control inputs to the same neuron. In cells that received control afferent stimulation (three per minute), phalloidin labeling was present in the cell body, the proximal portions of the basal dendrites, and in some cases, the primary apical dendritic shaft (Fig. $1 B$ ). Notably, dense labeling was absent in the fine branches of the apical dendritic ramification. As shown, very low levels of background labeling were present with intracellular applications of rhodaminephalloidin (Fig. 1B). Examination of s. radiatum at higher magnification revealed the presence of scattered puncta with sizes and distributions corresponding to those of spine heads in some, but not all, control slices.
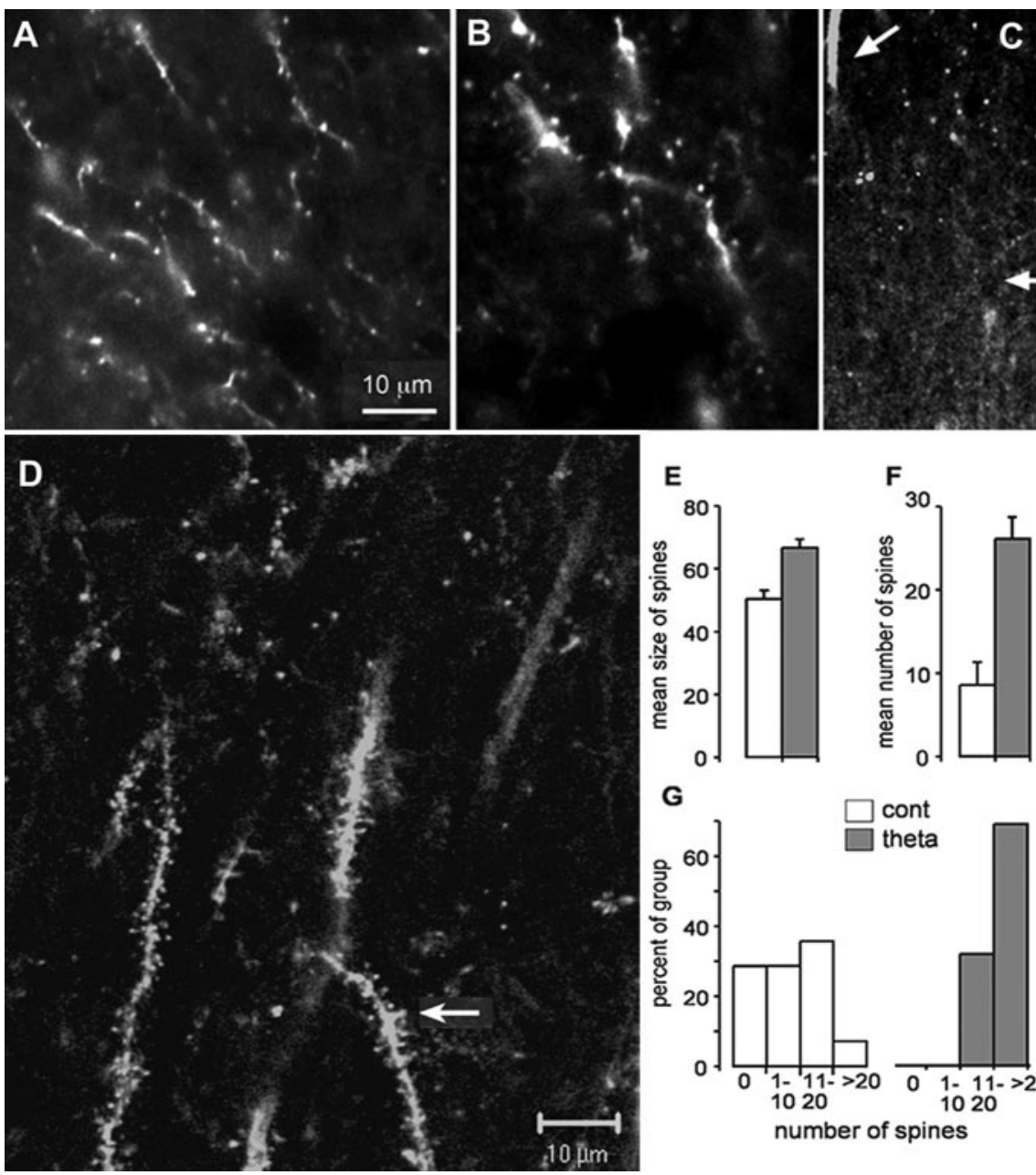

E

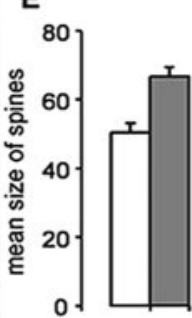

F

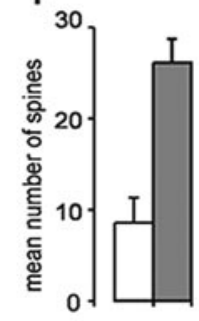

G

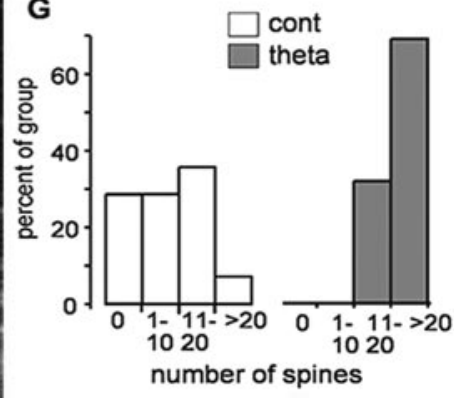

Figure 2. Theta bursts increase labeling of dendritic spines. $\boldsymbol{A}, \boldsymbol{B}$, Two cases, photographed at $40 \times$ objective magnification, in which densely labeled spine heads are located on stained segments of secondary dendritic branches. Note in $\boldsymbol{B}$ that the punctate at the top of the figure (top arrow). A small cluster of labeled spines is located to the right of the branch; the distribution of 列 . difference is highly significant $(p<0.005)$. $F$ Number of a Distribution of spine numbers across slices in the control and experimental groups. As shown, $<10 \%$ of the control slices had 10 or more labeled spines in the proximal half of s. radiatum, whereas $>60 \%$ of the theta burst cases fell into this category.

Theta bursts produced a dramatic increase in spine labeling in the apical dendritic field between the two stimulating electrodes, with the effect being primarily restricted to the inner half of $s$. radiatum. Although it cannot be stated with certitude that the labeled structures were apposed by stimulated terminals, current-source density analyses have shown that stimulating electrodes positioned as in the present study preferentially activate synapses in the lamina in which the puncta were located (Shimono et al., 2002). The labeled structures usually occurred in subregions, or neighborhoods, rather than being homogeneously distributed across the innervated zone. Figure $2 A-D$ shows representative cases. Note that labeled puncta often occur in conjunction with lightly labeled dendrites (Fig. $2 A, B$ ) but were also found in isolation, without staining of underlying processes, as illustrated in Figure 2C. In that micrograph, a small collection of probable spine heads can be seen below and to the right of the 

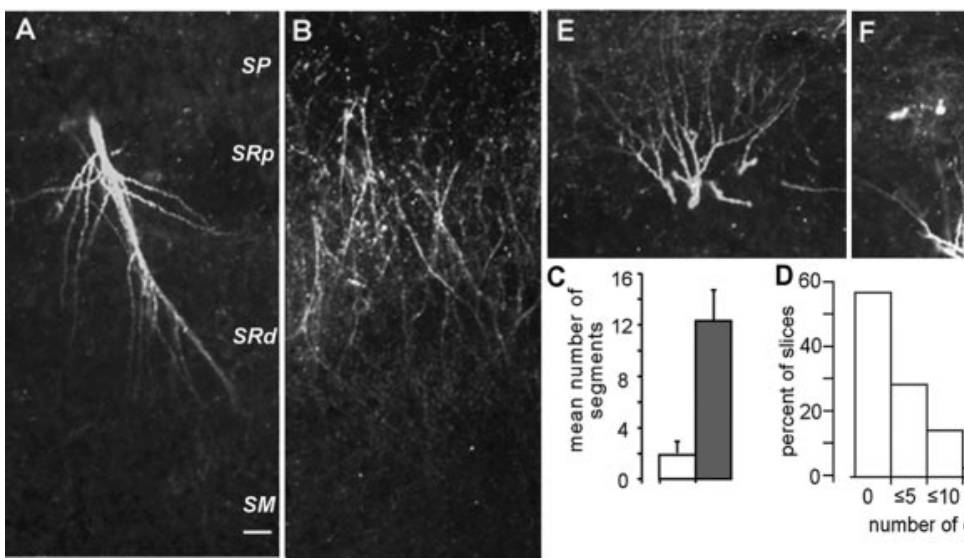

Figure 3. Theta burst stimulation causes actin polymerization in dendritic segments. $A, A$ primary (apical) dendritic branch from a cell with potentiated synapses. Note the numerous fine oblique dendritic branches. SP, Stratum pyramidale; SRp, proximal stratum radiatum; SRd, distal stratum radiatum; SM, stratum lacunosum-moleculare. $\boldsymbol{B}, A$ cluster of labeled segments from a theta burst slice in which multiple cells had been clamped. Note that the labeled segments are restricted to the inner half of the s. radiatum, the area innervated by the fibers that had received theta bursts. $\boldsymbol{C}$, Mean number of labeled dendritic segments in control (cont) and theta-stimulated (theta) slices; the difference between groups is highly significant ( $p=0.001)$. Error bars represent SEM. D, Distribution of numbers of stained segments across control and theta burst slices (bars represent numbers of segments per slice sample field, in nonoverlapping categories). Slightly $<40 \%$ of the potentiated slices did not have more labeled branches than found in controls; this value is higher than that for labeled spines (Fig. 2G). $\boldsymbol{E}, \boldsymbol{F}$, Labeled basal dendrites in two cells expressing LTP; $s$. pyramidale is at the bottom of each micrograph. Photographs were taken at $10 \times$ objective magnification. Scale bar: (in $\boldsymbol{A}) \boldsymbol{A}, \boldsymbol{B}, \boldsymbol{E}, \boldsymbol{F}, 20 \mu \mathrm{m}$.

primary shaft of the apical dendrite (arrow) with no evident labeling of the fine oblique branches of the tree; labeled spines are not present in the more distal segments of s. radiatum (small arrow marks the mid proximodistal point of the lamina). In confocal images, a number of the densely stained spine heads could be seen to have a thin, poorly stained neck attached to a fine dendritic process (Fig. 2D). Several spines that occur in isolation, not in association with a labeled dendritic segment, can be seen at the top of the image. Computerized counting of spines in a sampling zone $\left(5300 \mu \mathrm{m}^{2}\right)$ in proximal s. radiatum indicated that theta bursts increased the mean spine counts by a factor of three ( $p<0.0001 ; t$ test, two-tailed, here and afterward) (Fig. $2 F)$. The majority of control slices had 10 or fewer spines in the sampling zone, whereas over one-half of the theta burst slices had $>20$ (Fig. $2 G)$. The mean size of the spine heads was also greater $(34 \%)$ in the LTP cases than in controls ( $p=0.005$ ) (Fig. $2 E$ ), but whether this reflects an actual change in morphology as opposed to increased concentrations of F-actin could not be determined from the present material.

There were no evident differences in numbers of spines in slices collected at $2 \mathrm{~min}(22.5 \pm 5.3$ spines $)$ or $30 \mathrm{~min}(27.8 \pm$ $13.2 ; p>0.2)$ after TBS, but numbers in both groups were significantly higher than in controls.

Theta stimulation also caused an evident increase in the incidence and intensity of labeling in fine dendritic branches in several slices. Figure $3 A$ from a theta burst slice shows a primary dendrite with multiple oblique, fine processes. Similar labeled processes were not observed in 14 control (or seven pharmacological control; see below) experiments. Figure $3 B$ illustrates a case in which multiple cells had been clamped (i.e., cells were held for 15-30 min and then rejected for prolonged physiological recording) before delivery of theta bursts.

Note that the labeled dendritic segments are restricted to the middle and inner portion of s. radiatum, the lamina innervated by the stimulated fibers, and are not found in the more distal laminas above the target dendritic zone. Results from computer-

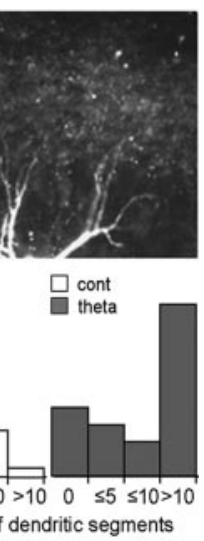

ized counting of labeled processes are summarized in Figure 3, $C$ and D. Labeled segments were virtually absent in control slices $(1.7 \pm 3.4$; mean $\pm \mathrm{SD})$ but were a common feature in theta burst slices $(12.3 \pm 10.5)$, a difference that was highly significant $(p=0.001)$. It is noteworthy that, unlike the results for spines, a significant number of theta burst slices $(37 \%$ of the group) did not have a sizeable population of labeled dendritic segments (Fig. $3 D)$. The difference in the theta burst slices between the frequencies of little or no effect cases for spines versus dendritic segments was statistically significant $(p<$ 0.01; Fisher's exact test).

Surprisingly, in a significant number of slices, theta bursts caused an increase in spine and dendritic segment labeling in s. oriens, a lamina that is not innervated by the apical wing of the Schaffercommissural projections. Figure 3, E and $F$, shows two examples from separate experiments. Much of the basal arbor is labeled in the case shown in Figure $3 E$, something that was not seen in the control slices. Spine heads were also in evidence but were not as large, or perhaps as intensely labeled, as was the case for the apical dendrites. In some instances, spines were present without strong labeling of the underlying fine dendritic processes (Fig. $3 F$ ). Quantitative analysis confirmed that there were substantially more labeled dendritic segments in s. oriens in slices that had received theta bursts than was the case for controls ( $3.5 \pm 3.0$ vs $0.5 \pm 0.7$ elements, respectively; $p=0.002)$.

We used two procedures to test whether the collection of TBSinduced changes in phalloidin labeling was associated with the induction of LTP or instead a consequence of intense synaptic activity. Infusing the NMDA receptor antagonist APV into the slices for 15 min before delivering theta bursts $(n=4)$ (Fig. $4 A$ ), or clamping the membrane potential at $-80 \mathrm{mV}$ during the bursts $(n=3)$ (Fig. $4 B$ ), blocked the induction of LTP (data not shown) and as shown in the low-power photomicrographs in Figure $4, A$ and $B$, had no evident effect on cell body and proximal dendritic labeling; that is, labeling was as intense as in control cells (Fig. $1 B$ ). Quantitative analysis at a higher magnification, more appropriate for visualizing spines, showed that blocking LTP eliminated any evidence of theta-induced actin polymerization $(1.6 \pm 2.3$ spines in the sampling area vs $26.1 \pm 11.4$ in LTP slices; $p=0.0001)$.

Finally, we tested whether extracellular applications of phalloidin could be used to check the results obtained with intracellular applications. Pilot studies indicated that $6 \mu \mathrm{M}$ concentrations of phalloidin could be applied topically to slices from young adult rats $(30-45 \mathrm{~d})$ for $20 \mathrm{~min}$ without evident disturbance of baseline physiology or LTP. Additional work showed that these infusions led to detectable labeling in slices that had been maintained for $>2 \mathrm{~h}$ in the chamber before topical application of phalloidin. Figure $5 \mathrm{~A}$ shows typical extracellular traces, collected from a single recording electrode in s. radiatum of field CA1b. The two pairs of fEPSPSs (S1 and S2) were elicited by current pulses to separate stimulating electrodes. The theta train stimulation via electrode S1 had no detectable effect on the baseline response, recorded $2 \mathrm{~min}$ later, to stimulation pulses delivered to 


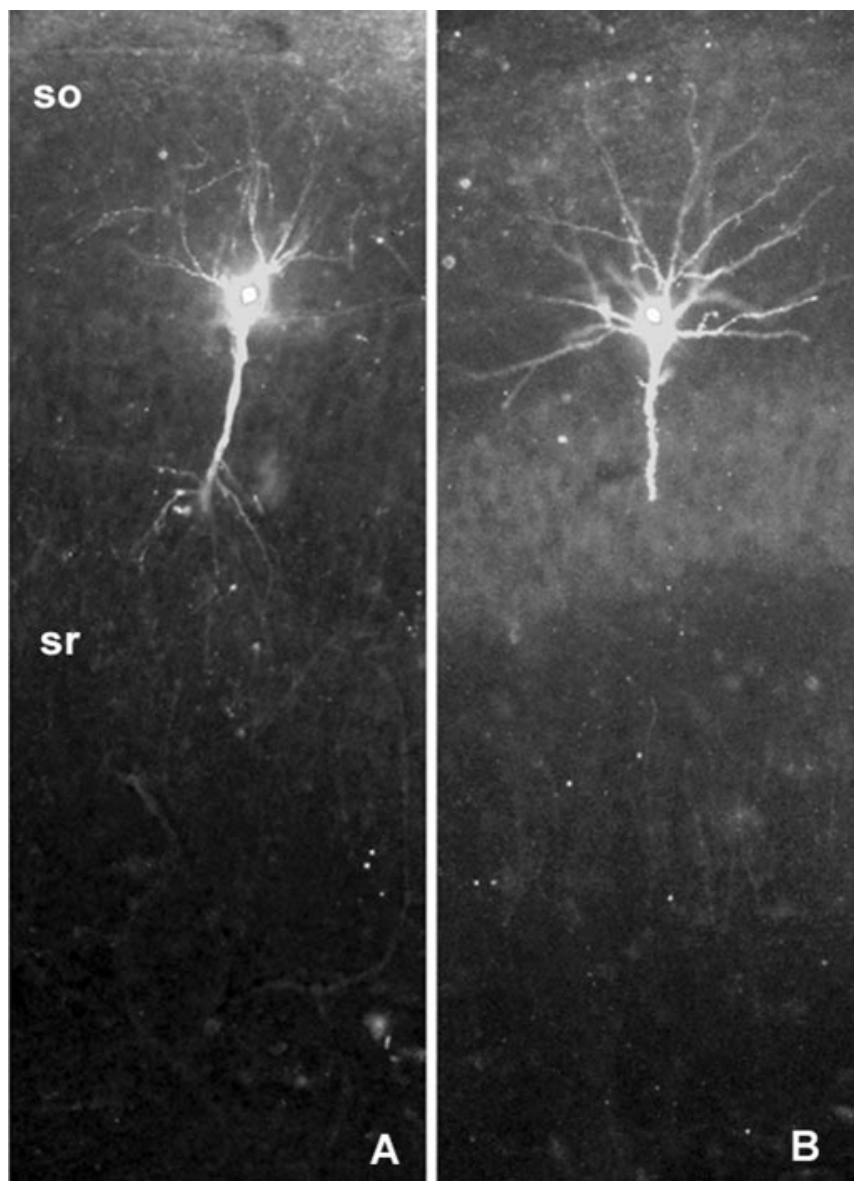

Figure 4. Blocking LTP induction does not prevent the labeling of cell bodies and primary dendritic branches. The rhodamine-phalloidin-labeled cell to the left $(\boldsymbol{A})$ is from a slice that had been infused with the NMDA receptor antagonist APV from $15 \mathrm{~min}$ before the delivery of theta bursts to its Schaffer-commissural afferents, whereas the labeled cell on the right $(\boldsymbol{B})$ was clamped at $-80 \mathrm{mV}$ during the theta burst train. There was no evidence of increased spine staining in these cases, although, as shown, labeling of the cell bodies and primary dendritic branches was robust. so, Stratum oriens; sr, stratum radiatum. Magnification, $10 \times$.

electrode S2, thereby showing that the stimulation conditions did not produce heterosynaptic effects. The magnitude of the homosynaptic, short-term potentiation produced by theta trains was equivalent to that described in previous studies.

Slices were fixed $1 \mathrm{~min}$ after delivery of a theta train to electrode S2. There was very little background staining in control slices (Fig. 5B,D), and puncta corresponding in size and density to spine heads were infrequent (Fig. 5D). Theta burst stimulation produced a dramatic increase in the number of labeled spine heads (Figs. 5C,E). Labeling of dendritic branches (Fig. 5C) was uncommon, and spines were typically found in isolation or associated with thin, faintly labeled segments (Fig. 5E). Quantitative analysis indicated that theta bursts caused a $>10$-fold increase in the number of labeled spine heads in the sampling zone $(p<$ 0.002) (Fig. 5F). This effect was substantially greater than that obtained with intracellular labeling. The effects on spine number were not accompanied by an evident increase in the low numbers of labeled dendritic segments (Fig. $5 F$ ). Importantly, unlike the case for intracellular labeling, there was very little labeling in the basal dendrites, and this was not detectably affected by theta stimulation of s. radiatum afferents. Additional experiments using the extracellular version of in situ labeling showed that the increased spine labeling produced by theta bursts is persistent. Figure 6 shows the sampling region in the inner stratum radiatum for three slices ("TBS a,b,c") that were fixed 30 min after receiving theta burst stimulation; the increase in stained puncta over a typical control (left panel) is apparent. The effects of theta stimulation on phalloidin labeling were substantially reduced by previous infusion of APV (right panel) at a concentration $(100 \mu \mathrm{M})$ that prevented the induction of LTP (Fig. 6, bottom for summary of physiology). Quantitative comparisons confirmed that labeled puncta were much more frequent $30 \mathrm{~min}$ after theta stimulation (34.4 $\pm 7.4 ; n=5$ slices) than was the case in slices given only control stimulation $(1.3 \pm 0.6 ; n=3$ slices; $p<0.0006$ for $t$ test with unequal variances). These control and experimental values are close to those obtained with very short delays after theta bursts (Fig. 5F). Labeling in the theta burst plus APV-treated slices (11.2 $\pm 14.3 ; n=5$ slices), although variable, was clearly less than in the LTP group $(p<0.02)$ and not significantly different from controls $(p>0.2)$. Finally, as in the first extracellular application study, there was no evidence of basal dendritic staining in the experiment just described.

\section{Discussion}

Chronic recording studies indicate that LTP can persist for several weeks with little evident change in magnitude (Staubli and Lynch, 1987; Abraham et al., 2002). This longevity in the face of protein turnover suggests, although does not require, that the substrates of LTP involve anatomical changes. Spines are attractive candidates for the locus of such effects because of their (1) recently demonstrated stability (Grutzendler et al., 2002; Trachtenberg et al., 2002) and (2) intimate relationship to the postsynaptic specialization in which transmitter receptors are located. Regarding the latter, recent work indicates that the size of the postsynaptic apposition zone correlates with the number of AMPA-type glutamate receptors it contains (Nusser et al., 1998; Takumi et al., 1999). Rounding or segmenting of spines could therefore enhance the synaptic response simply by increasing the area of the postsynaptic density and thus the size of the receptor pool. Combining these points, changes in spine shape, as described in experiments using electron microscopy (Yuste and Bonhoeffer, 2001) or light microscopy (Matsuzaki et al., 2004), constitute plausible explanations for both the stability and the expression of LTP.

Shape change in cells or their processes, over a diverse array of experimental conditions, requires reorganization of the actin cytoskeleton. Spines contain significant concentrations of actin (Matus et al., 1983), and high-frequency synaptic activity results in rapid polymerization of spine actin in dissociated neurons (Ackermann and Matus, 2003). Intense afferent stimulation also causes actin polymerization in spines in organotypic cultures (Okamoto et al., 2004) and throughout the full extent of the dentate gyrus outer molecular layer in vivo (Fukazawa et al., 2003). Using a different technology, we now show that a single train of theta bursts, a degree of stimulation only slightly greater than the threshold for inducing stable LTP (Larson et al., 1986), rapidly polymerizes actin in discrete populations of spines and that this effect is readily obtained from late development through early adulthood. Under the described conditions, the method of in situ labeling with phalloidin to follow actin assembly (Ma et al., 1998), which has not been previously described for neurons, causes no evident disturbances of physiology. The extracellular variant of the technique, other than apparently requiring the use of slices maintained for hours, is particularly straightforward and should prove useful in studies of the mechanisms regulating actin polymerization in adult spines. 

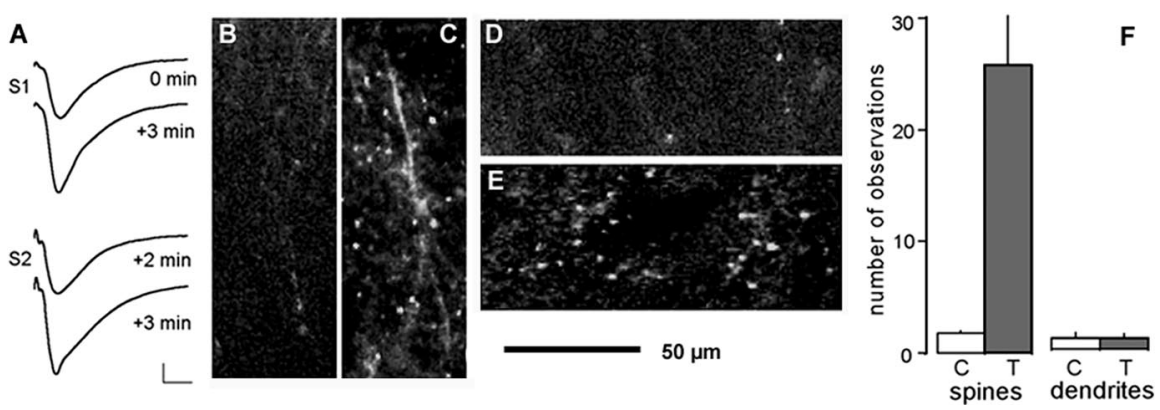

Figure 5. Effects of theta burst stimulation on labeling after topical application of rhodamine-phalloidin. $\boldsymbol{A}$, Field EPSPS recorded at a single site in s. radiatum of CA1b and elicited by stimulating electrodes in CA1a (S1) or CA1c (S2). Theta bursts were delivered to $\mathrm{S} 1$ and then $2 \mathrm{~min}$ later to $\mathrm{S2}$. The induction of short-term potentiation in $\mathrm{S} 1 \mathrm{had}$ no evident effect on the response in S2 recorded $2 \mathrm{~min}$ later. Calibration bars: $0.5 \mathrm{mV}, 5 \mathrm{~ms} . \boldsymbol{B}, \boldsymbol{D}$, Labeling in the standard-sized sampling zone placed over areas of afferent stimulation in the proximal s. radiatum of two control slices. $C, E$, Labeling in fields comparable with those shown in $B$ and $D$ in two slices that had received theta burst stimulation. A fine dendritic segment is labeled in $C$; similar processes are very faint or not labeled in $E$. $F$, Number of labeled spines and dendritic branches in the sampling zone in slices given control $(C ; n=4)$ or theta burst $(\mathrm{T} ; n=5)$ stimulation. Note that the large increase $(\sim 14$-fold; $p<0.002)$ in phalloidin-positive spines was elicited by the latter treatment without any evident increase in labeled dendritic segments. Magnification, $20 \times$. Scale bar: $\boldsymbol{B}-\boldsymbol{E}, 50 \mu \mathrm{m}$. Error bars represent SEM.
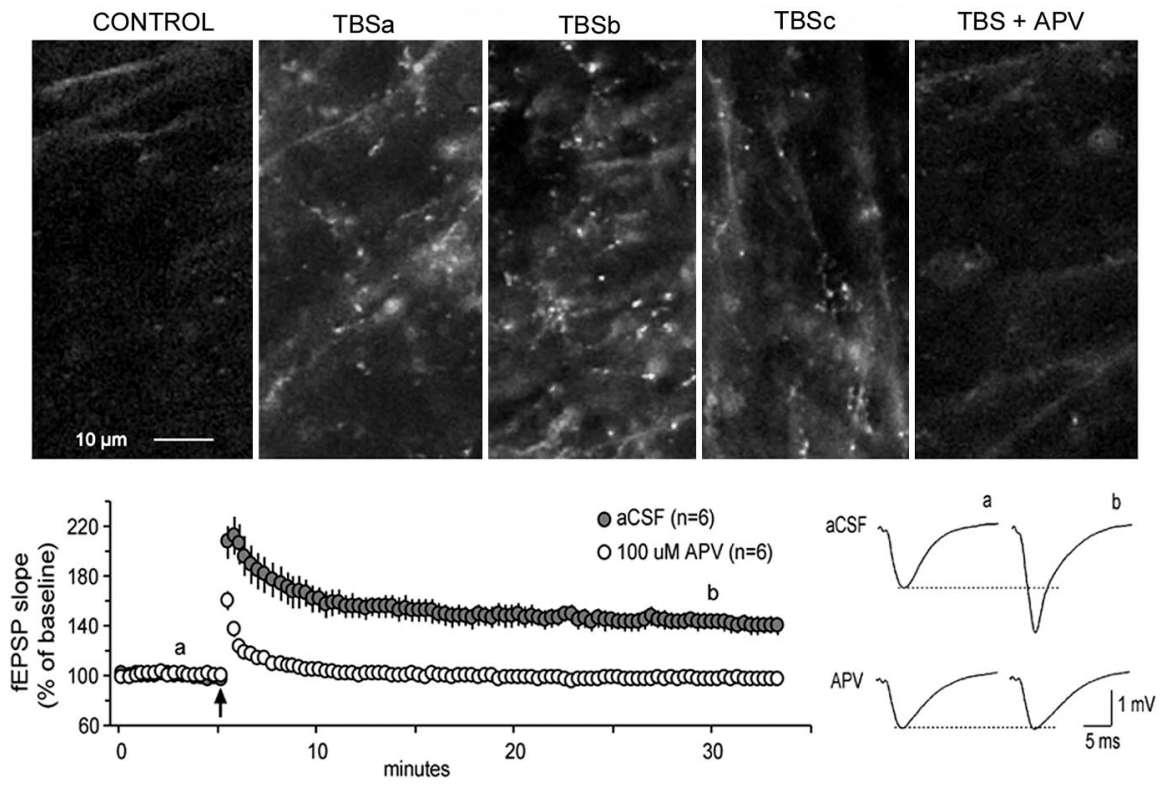

Figure 6. Theta stimulation-induced increases in rhodamine-phalloidin labeling persist for at least $30 \mathrm{~min}$ and are blocked by the NMDA receptor antagonist APV in slices from young adult rats. Photomicrographs of labeling within the sampling zone in stratum radiatum after topical application of rhodamine-phalloidin are shown; stimulating and recording electrodes were located as described in Figure 5. Top panels (from left to right) show the sampling zone in a control slice, in three slices $(a, b, c)$ that received TBS, and in a fourth slice in which TBS was delivered in the presence of $100 \mu \mathrm{m}$ APV. The slices were fixed 30 min after theta burst or (for control) low-frequency stimulation. As shown, relative to control, there was a large increase in punctate labeling with TBS stimulation but not with TBS plus APV. The graph at the bottom describes the effects of theta burst stimulation on the group mean \pm SEM of the initial slopes of fEPSPs recorded from the proximal s. radiatum and elicited by stimulation of the Schaffercommissural projections. Traces are representative fEPSP recordings from ACSF- and APV-infused slices that were collected during the period of baseline recordings (a) and 30 min after theta burst stimulation (b). Both the graph and traces demonstrate that APV completely blocked the induction of LTP.

Numerous studies have shown that induction of LTP in a small population of inputs to a dendritic region leaves unaffected the remaining, much larger population of synapses in that region (Andersen et al., 1977; Lynch et al., 1977). This synapsespecificity rule, which is critical to arguments about the role of LTP in memory, requires that candidate substrates of the potentiation effect engage only a small percentage of contacts in any given dendritic region. The increases in phalloidin-positive spines found in the present study appear to satisfy this rule. That

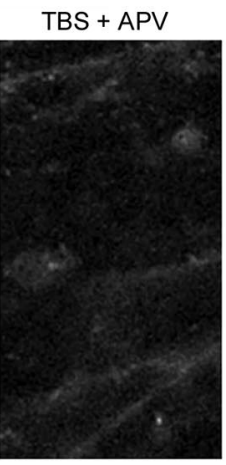

is, although the effect was large relative to control slices, it involved only a small fraction of the spine population (i.e., for the whole-cell study, approximately five spines per $100 \mu \mathrm{m}^{2}$ were labeled in a field in which there are 4.9 spines per micrometer of segment length) (Kirov et al., 1999). It can be assumed that the numerous segments arising from recorded cells add up to hundreds of micrometers, so that unlabeled spines on the target neuron far outnumber the labeled instances. In addition to falling within the constraints of the synapse-specificity rule, the small number of labeled spines is consistent with the argument that modest-sized composite EPSCs $(\sim 70 \mathrm{pA})$ recorded at cell bodies in hippocampus are generated by $<50$ contacts (Hanse and Gustafsson, 2001). There were large (14-fold) increases in phalloidin-positive spines in the experiments using extracellular applications, but, in this case as well, the absolute numbers involved were a small fraction of the number of spines found within a 5300 $\mu \mathrm{m}^{2}$ region of s. radiatum (Kirov et al., 1999; Shi et al., 2005).

However, two aspects of the whole-cell study suggest that the stimulation conditions used in our experiments were near a borderline for causing changes that extend beyond the synaptic region. Polymerization (1) was seen in numerous fine branches within the proximal s. radiatum and (2) occurred in the basal dendrites in a subset of slices. Although the latter effect could reflect a nonspecific effect of depolarization or action potential invasion, the NMDA receptor antagonist APV, which blocks only a portion of the depolarization produced by theta bursts (Larson and Lynch, 1988), completely eliminated the increases in phalloidin staining in the basal as well as apical dendrites. An alternative possibility is that the polymerization was triggered by activation of axon collaterals arising from the target pyramidal cells (Alger and Teyler, 1977; Amaral et al., 1991). Importantly, theta burst stimulation did not increase the numbers of labeled apical or basal dendrites in the extracellular recording study, indicating that these effects depend on the manner in which the slice is maintained (submerged vs interface) and, perhaps, the techniques used to induce potentiation (theta bursts alone in the field potential studies vs theta bursts and depolarization in the clamp experiments). A challenge for future studies will be to compare the threshold conditions for inducing LTP with those that induce actin polymerization in spine heads.

Past studies have shown that LTP, like memory, undergoes progressive stabilization starting $30 \mathrm{~s}$ after induction and continuing for the next 30-60 min (Barrionuevo et al., 1980; Lynch et al., 1991; Staubli and Chun, 1996; C. C. Huang et al., 2001). 
Other work points to the existence of a distinct, protein synthesisdependent period of consolidation that begins hours later (Nguyen et al., 1994; Bradshaw et al., 2003). The question thus arises of whether actin polymerization is rapid enough to be an important contributor to the process that causes LTP to become resistant to disruption over the first few minutes after its induction. The increase in labeled spines was evident within 2 min of the theta bursts in the whole-cell recording study, the earliest postinduction time point tested. Moreover, the magnitude of the change was as large at that time point as it was $30 \mathrm{~min}$ after induction. The increases in spine labeling obtained with topical applications also were very rapid (1-3 min) and again persisted for at least $30 \mathrm{~min}$. These results suggest that polymerization must be initiated almost immediately and reaches what may be a near-maximum level within $2 \mathrm{~min}$. This is extremely rapid but plausible; for example, actin polymerization in Xenopus egg extracts is well advanced within $60 \mathrm{~s}$ of adding an appropriate stimulus (Ma et al., 1998). We cannot determine from the present results whether actin polymerization is part of a new spine state or instead contributes to a transient process that results in a new and stable configuration. The former idea does not seem likely given that slices contain a wide array of spine types but appear to have very few spines with the concentrations of polymerized actin found after LTP induction. Still, experimental results on the duration of the polymerization induced by theta bursts could prove valuable in developing hypotheses about the still poorly understood process whereby spines change their shapes.

Finally, there remains the question of how afferent activity causes the above effects. Rapid polymerization of actin in developing or motile cells is generally triggered by integrins, but there appear to be no studies testing whether the adhesion receptors have such signaling capability in terminally differentiated neurons in adult brain. The issue is particularly relevant to the present results because of a growing literature implicating integrins in the consolidation of LTP. Integrin ligands of different types block consolidation (Staubli et al., 1990, 1998; Xiao et al., 1991; Bahr et al., 1997) and do so with decreasing efficiency over the 30 min after LTP induction (Staubli et al., 1998). Experiments using function blocking antibodies identified the $\alpha 3 \beta 1$ and $\alpha 5 \beta 1$ dimers as being among the pertinent integrins (Chun et al., 2001; Kramár et al., 2002; Kramár and Lynch, 2003), and results from transgenic mouse studies are in agreement with this conclusion (Chan et al., 2003): LTP consolidation, but not induction, was substantially impaired in animals with both $\alpha 3+/-$ and $\alpha 5+/-$ mutations and essentially absent in triple transgenics $(\alpha 3, \alpha 5$, and $\alpha 8$ heterozygotes). Integrins exert their intracellular effects, in part, by activating the homologous tyrosine kinases FAK (focal adhesion kinase) and Pyk2 (proline-rich tyrosine kinase 2) (Vuori, 1998), and suppressing either of these enzymes prevents the stabilization of LTP (Y. Huang et al., 2001; Yang et al., 2003). The results described here lead to the testable hypothesis that integrins exert their effects on LTP via their potent influence over the actin cytoskeleton.

\section{References}

Abraham WC, Logan B, Greenwood JM, Dragunow M (2002) Induction and experience-dependent consolidation of stable long-term potentiation lasting months in the hippocampus. J Neurosci 22:9626-9634.

Ackermann M, Matus A (2003) Activity-induced targeting of profilin and stabilization of dendritic spine morphology. Nat Neurosci 6:1194-1200.

Alger BE, Teyler T (1977) A monosynaptic fiber track studied in vitro: evidence of a hippocampal CA1 associational system? Brain Res Bull 2:355-365.

Amaral DG, Dolorfo C, Alvarez-Royo P (1991) Organization of CA1 pro- jections to the subiculum: a PHA-L analysis in the rat. Hippocampus 1:415-435.

Andersen P, Sundberg SH, Sveen O, Wigstrom H (1977) Specific longlasting potentiation of synaptic transmission in hippocampal slices. $\mathrm{Na}$ ture 266:736-737.

Arai A, Black J, Lynch G (1994) Origins of the variations in long-term potentiation between synapses in the basal versus apical dendrites of hippocampal neurons. Hippocampus 4:1-9.

Bahr BA, Staubli U, Xiao P, Chun D, Ji Z-X, Esteban ET, Lynch G (1997) Arg-Gly-Asp-Ser-selective adhesion and the stabilization of long-term potentiation: pharmacological studies and the characterization of a candidate matrix receptor. J Neurosci 17:1320-1329.

Barrionuevo G, Schottler S, Lynch G (1980) The effects of repetitive low frequency stimulation on control and "potentiated" synaptic responses in the hippocampus. Life Sci 27:2385-2391.

Bliss TVP, Lomo T (1973) Long-lasting potentiation of synaptic transmission in the dentate area of the anesthetized rabbit following stimulation of the perforant path. J Physiol (Lond) 232:334-356.

Bradshaw KD, Emptage NJ, Bliss TVP (2003) A role for dendritic protein synthesis in hippocampal late LTP. Eur J Neurosci 18:3150-3152.

Chan CS, Weeber EJ, Kurup S, Sweatt JD, Davis RL (2003) Integrin requirement for hippocampal synaptic plasticity and spatial memory. J Neurosci 23:7107-7116.

Chang FL, Greenough WT (1984) Transient and enduring morphological correlates of synaptic activity and efficacy change in the rat hippocampal slice. Brain Res 309:35-46.

Chun D, Gall CM, Bi X, Lynch G (2001) Evidence that integrins contribute to multiple stages in the consolidation of long term potentiation. Neuroscience 105:815-829.

Desmond NL, Levy WB (1986) Changes in the postsynaptic density with long-term potentiation in the dentate gyrus. J Comp Neurol 253:476-482.

Fiala JC, Allwardt B, Harris KM (2002) Dendritic spines do not split during hippocampal LTP or maturation. Nat Neurosci 5:297-298.

Fischer M, Kaech S, Knutti D, Matus A (1998) Rapid actin-based plasticity in dendritic spines. Neuron 20:847-854.

Fukazawa Y, Saitoh Y, Ozawa F, Ohta Y, Mizuno K, Inokuchi K (2003) Hippocampal LTP is accompanied by enhanced F-actin content within the dendritic spine that is essential for late LTP maintenance in vivo. Neuron 38:447-460.

Geinisman Y, DeToledo-Morrel L, Morrel F (1991) Induction of long-term potentiation is associated with an increase in the number of axospinous synapses with segmented postsynaptic densities. Brain Res 566:77-88.

Grutzendler J, Kasthuri N, Gan WB (2002) Long-term dendritic spine stability in the adult cortex. Nature 420:751-752.

Hanse E, Gustafsson B (2001) Quantal variability at glutamatergic synapses in area CA1 of the rat neonatal hippocampus. J Physiol (Lond) 531:467-480.

Huang CC, Liang YC, Hsu KS (1999) A role for extracellular adenosine in time-dependent reversal of long-term potentiation by low-frequency stimulation at hippocampal CA1 synapses. J Neurosci 19:9728-9738.

Huang CC, Liang YC, Hsu KS (2001) Characterization of the mechanism underlying the reversal of long term potentiation by low frequency stimulation at hippocampal CAl synapses. J Biol Chem 276:48108-48117.

Huang Y, Lu WY, Ali DW, Pelkey KA, Pitcher GM, Lu YM, Aoto H, Roder JC, Saski T, Salter MW, MacDonald JF (2001) CAK $\beta / P Y K 2$ kinase is a signaling link for induction of long-term potentiation in CA1 hippocampus. Neuron 29:485-496.

Jain A (1989) Fundamentals of digital image processing. Englewood Cliffs, NJ: Prentice-Hall.

Kirov SA, Sorra KE, Harris KM (1999) Slices have more synapses than perfusion-fixed hippocampus from both young and mature rats. J Neurosci 19:2876-2886.

Kramár EA, Lynch G (2003) Developmental and regional differences in the consolidation of long-term potentiation. Neuroscience 118:387-398.

Kramár EA, Bernard JA, Gall CM, Lynch G (2002) Alpha3 integrin receptors contribute to the consolidation of long-term potentiation. Neuroscience 110:29-39.

Krucker T, Siggins GR, Halpain S (2000) Dynamic actin filaments are required for stable long-term potentiation (LTP) in area CA1 of the hippocampus. Proc Natl Acad Sci USA 97:6856-6861. 
Larson J, Lynch G (1988) Role of N-methyl-D-aspartate receptors in the induction of synaptic potentiation by burst stimulation patterned after the hippocampal theta-rhythm. Brain Res 441:111-118.

Larson J, Wong D, Lynch G (1986) Patterned stimulation at the theta frequency is optimal for the induction of hippocampal long-term potentiation. Brain Res 368:347-350.

Larson J, Xiao P, Lynch G (1993) Reversal of LTP by theta frequency stimulation. Brain Res 600:97-102.

Lee KS, Schottler F, Oliver M, Lynch G (1980) Brief bursts of highfrequency stimulation produce two types of structural change in rat hippocampus. J Neurophysiol 44:247-258.

Lin B, Brucher FA, Colgin LL, Lynch G (2002) Long-term potentiation alters the modulator pharmacology of AMPA-type glutamate receptors. J Neurophysiol 87:2790-2800.

Lynch G, Bahr BA, Vanderklish PW (1991) Induction and stabilization of LTP. In: Glutamate, cell death and memory (Ascher P, Choi DW, Christen Y, eds), pp 45-60. Berlin: Springer.

Lynch GS, Dunwiddie T, Gribkoff V (1977) Heterosynaptic depression: a postsynaptic correlate of long-term potentiation. Nature 266:737-739.

Ma L, Rohatgi R, Kirschner MW (1998) The Arp2/3 complex mediates actin polymerization induced by the small GTP-binding protein Cdc42. Proc Natl Acad Sci USA 95:15362-15367.

Matsuzaki M, Honkura N, Ellis-Davies GC, Kasai H (2004) Structural basis of long-term potentiation in single dendritic spines. Nature 429:761-766.

Matus A (2000) Actin-based plasticity in dendritic spines. Science 290:754-758.

Matus A, Ackermann M, Pehling G, Byers HR, Fujiwara K (1983) High actin concentrations in brain dendritic spines and postsynaptic densities. Proc Natl Acad Sci USA 79:7590-7594.

Nguyen PV, Abel T, Kandel ER (1994) Requirement of a critical period of transcription for induction of a late phase of LTP. Science 265:1104-1107.

Nusser Z, Lujan R, Laube G, Roberts JD, Molnar E, Somogyi P (1998) Cell type and pathway dependence of synaptic AMPA receptor number and variability in the hippocampus. Neuron 21:545-559.

Okamoto K-I, Nagai T, Miyawaki A, Hayashi Y (2004) Rapid and persistent modulation of actin dynamics regulates postsynaptic reorganization underlying bidirectional plasticity. Nat Neurosci 7:1104-1112.
Shi L, Linville MC, Tucker EW, Sonntag WE, Brunso-Bechtold JK (2005) Differential effects of aging and insulin-like growth factor-1 on synapses in CA1 of rat hippocampus. Cereb Cortex, in press.

Shimono K, Kubota D, Brucher F, Taketani M, Lynch G (2002) Asymmetrical distribution of the Schaffer projections within the apical dendrites of hippocampal field CA1. Brain Res 950:279-287.

Staubli U, Chun D (1996) Factors regulating the reversibility of long-term potentiation. J Neurosci 16:853-860.

Staubli U, Lynch G (1987) Stable hippocampal long-term potentiation elicited by "theta" pattern stimulation. Brain Res 435:227-234.

Staubli U, Chun D, Lynch G (1998) Time-dependent reversal of long-term potentiation by an integrin antagonist. J Neurosci 18:3460-3469.

Staubli U, Vanderklish PW, Lynch G (1990) An inhibitor of integrin receptors blocks LTP. Behav Neural Biol 53:1-5.

Takumi Y, Ramirez-Leon V, Laake P, Rinvik E, Ottersen OP (1999) Different modes of expression of AMPA and NMDA receptors in hippocampal synapses. Nat Neurosci 2:618-624.

Toni N, Buchs PA, Nikonenko I, Bron CR, Muller D (1999) LTP promotes formation of multiple spine synapses between a single axon terminal and a dendrite. Nature 402:421-425.

Trachtenberg JT, Chen BE, Knott GW, Feng G, Sanes JR, Welker E, Svoboda $\mathrm{K}$ (2002) Long-term in vivo imaging of experience-dependent synaptic plasticity in adult cortex. Nature 420:788-794.

Van Harreveld A, Fifkova E (1975) Swelling of dendritic spines in the fascia dentata after stimulation of the perforant fibers as a mechanism of posttetanic potentiation. Exp Neurol 49:736-749.

Vuori K (1998) Integrin signaling: tyrosine phosphorylation events in focal adhesions. J Membr Biol 165:191-199.

Xiao P, Bahr BA, Staubli U, Vanderklish PW, Lynch G (1991) Evidence that matrix recognition contributes to stabilization but not induction of LTP. NeuroReport 2:461-464.

Yang YC, Ma YL, Chen SK, Wang CW, Lee EHY (2003) Focal adhesion kinase is required, but not sufficient, for the induction of long-term potentiation in dentate gyrus neurons in vivo. J Neurosci 23:4072-4080.

Yuste R, Bonhoeffer T (2001) Morphological changes in dendritic spines associated with long-term synaptic plasticity. Annu Rev Neurosci 24:1071-1089. 